\title{
Avaliação bayesiana das incertezas nas estimativas dos parâmetros de um modelo chuva-vazão conceitual
}

\author{
Francisco Eustáquio Silva \\ Universidade Federal de Minas Gerais; Potamos Engenharia e Hidrologia - fcoeustaquio@gmail.com
}

Mauro Naghettini e Wilson Fernandes

Universidade Federal de Minas Gerais-naghet@netuno.lcc.ufmg.br; wilson@ehr.ufmg.br

Recebido: 02/12/13 - revisado: 04/02/14 - aceito: 27/06/14

\begin{abstract}
RESUMO
Neste artigo são avaliadas as incertezas associadas às estimativas do conjunto de parâmetros de um modelo chuva-vazão conceitual, por meio do emprego de técnicas de inferência bayesiana via simulações de Monte Carlo. Uma sub-bacia do Rio Pará, localizada na parte alta da bacia do Rio São Francisco, foi selecionada para o desenvolvimento dos estudos. Empregou-se na análise o modelo Rio Grande (EHR/UFMG, 2001) e o método de simulação de Monte Carlo via cadeias de Markov denominado DREAM (VRUGT, 2008a). Os resultados demonstraram que a abordagem bayesiana revelou-se adequada aos objetivos propostos, permitindo e reforcando a importância de se avaliarem as incertezas associadas aos resultados das simulaçoes por modelos hidrológicos.
\end{abstract}

Palavras Chave: Modelagem chuva-vazão. Avaliação de incertezas. Inferência bayesiana. Modelo Rio Grande. DREAM.

\section{INTRODUÇÃO}

O desenvolvimento e a avaliação de modelos que descrevem o processo de transformação da chuva em vazão têm sido o foco de diversas pesquisas realizadas nas últimas décadas (BURNASH et al., 1973 apud SINGH; WOOLHISER, 2002; ZHAO, 1992; LINDSTROM et al., 1997; MOORE, 2007). As razões para o desenvolvimento e aplicação destes modelos são diversas, conquanto podem ser sumarizadas pelas limitações das técnicas de medição das variáveis hidrológicas no espaço e no tempo (BEVEN, 2012). A utilização de modelos permite aos hidrólogos extrapolar o conhecimento adquirido por meio de observações, tanto em termos espaciais, particularmente úteis no caso de bacias não monitoradas, quanto temporais, quando o objetivo é realizar previsões dos estados futuros de variáveis associadas ao fenômeno modelado (vazão, volume, nível do curso d'água etc.).

Liu e Gupta (2007) destacam que o desenvolvimento destes modelos e o consequente aprimoramento da compreensão da física e dinâmica dos sistemas hidrológicos foram fortemente beneficiados pela crescente capacidade computacional, aliada a um aumento na disponibilidade de informações hidrológicas. Paradoxalmente, enquanto esses avanços refletiram uma maior compreensão do fenômeno, eles também consolidaram a necessidade do desenvolvimento de métodos mais robustos para avaliação das incertezas associadas aos prognósticos realizados por estes modelos.

De fato, a estimativa da incerteza na modelagem hidrológica é atualmente uma das áreas de pesquisa mais ativas em hidrologia (MONTANARI, 2011). Corroboram para esta observação o crescente número de publicações dedicadas a este tema nos últimos anos (KUCZERA; PARENT, 1998; VRUGT et al., 2003; WAGENER, et al., 2003; KUCZERA, et al., 2006; SIRORSKA et al., 2012; SUN; BERTRAND-KRAJWESKI, 2013). O presente artigo insere-se neste contexto e se propõe a avaliar as incertezas associadas às estimativas dos parâmetros de um modelo chuva-vazão de base conceitual, tal como aplicado à simulação hidrológica de uma sub-bacia do Rio São Francisco, no Estado de Minas Gerais.

O artigo foi organizado em 6 seções, além desta primeira (1) introdutória; na segunda seção (2) é apresentada uma contextualização do tema; na seção (3) a metodologia utilizada é descrita; os resultados obtidos são apresentados e analisados na seção (4); na seção (5) são realizadas as considerações finais do estudo; por fim, na última seção (6) as referências consultadas são apresentadas.

\section{ANÁLISE DE INCERTEZAS NA MODELAGEM CHUVA-VAZÃO}

A incerteza, no contexto da modelagem chuva-vazão, decorre da incompletude da compreensão do processo físico de transformação da chuva em vazão, associada à imperfeição das técnicas de medição utilizadas para obtenção das variáveis hidrológicas. Elas provêm essencialmente de três fontes (SHRESTHA, 2009):

Dados (input: $u$; e output:y): erros em $u$ (ex.: precipitação e evaporação), e em $y$ (ex.: vazão), ou ainda nas variáveis de estados iniciais $\left(x_{0}\right)$, quando as mesmas são obtidas a partir de observações de campo (ex.: teor de umidade do solo, nível de água no subsolo). Eles decorrem essencialmente de erros no processo de medição, seja por limitação dos instrumentos ou falha humana, e/ou da inadequada representatividade da amostra, em termos espaciais e temporais;

Estrutura $(M)$ e condições de contorno $(B)$ do modelo: modelos conceituais são apenas abstrações da realidade, as quais geralmente envolvem certo grau de simplificação e/ou idealização. Aproximações inapropriadas, ou mesmo desconsideradas, dos principais processos físicos podem resultar em erros significativos. Ademais, estes erros podem advir da implementação 
matemática (discretização espacial e temporal), a qual converte a estrutura conceitual em um modelo numérico;

Parâmetros $(\theta)$ e variáveis de estado $(x)$ : incertezas nos parâmetros e variáveis de estado derivam da inabilidade de obterem-se medidas precisas dos dados de entrada do modelo. Os parâmetros do modelo nem sempre possuem um significado físico direto. Por conseguinte, eles não podem ser medidos diretamente em campo ou o custo associado inviabiliza sua realização. Neste caso, os valores são estimados a partir de métodos indiretos (ex.: baseados na experiência do modelador, através da calibração dos parâmetros), os quais podem constituir-se em fontes adicionais de incerteza.

A Figura 1 apresenta esquematicamente os componentes e as possíveis fontes de incerteza da simulação hidrológica por um modelo chuva-vazão.

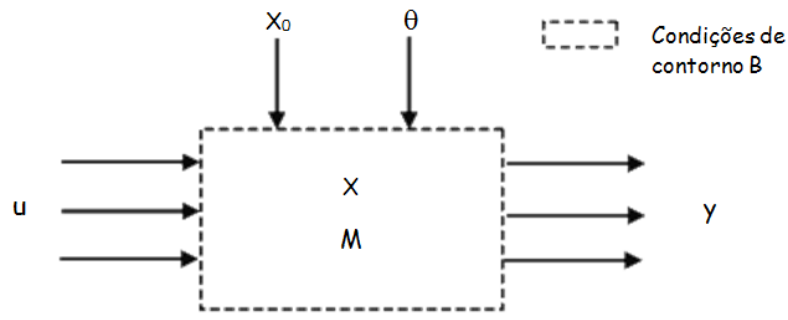

Figura 1 - Componentes e fontes de incerteza de um modelo (adaptado de LIU; GUPTA, 2007)

É necessário ponderar que, independentemente da fonte, existem duas categorias distintas de incerteza (BEVEN, 2009; GONG et al., 2013): (i) uma aleatória (ou intrínseca), cuja natureza reside na diferença observada nos resultados obtidos através da repetição de um experimento sob as mesmas condições; e (ii) outra epistêmica, que decorre da insuficiência ou ausência de compreensão e/ou informação sobre todos os processos físicos que governam o fenômeno modelado. A redução da incerteza epistemológica ocorre por meio da obtenção de informações complementares, aprimoramento dos modelos e/ou de uma melhor estimativa dos parâmetros e variáveis de estado. O presente artigo concentra-se nesta última categoria, mais especificamente nas incertezas associados aos parâmetros $\theta$ que integram a estrutura conceitual do modelo analisado.

Entre os diversos métodos utilizados para avaliação da incerteza, os denominados probabilísticos são, de longe, os mais tradicionais, bem compreendidos e mais testados (SHRESTHA, 2009). Segundo Paulino et al. (2003), há duas abordagens acerca do conceito de probabilidade: a frequentista ou clássica e a subjetiva ou bayesiana. Ambas compreendem, que as incertezas sobre as variáveis aleatórias devem ser mensuradas via probabilidades. No entanto, no contexto clássico, a medida de probabilidade capta a variabilidade inerente ao processo e, no contexto bayesiano, tal medida captura o desconhecimento do indivíduo sobre a variável em estudo. Como consequência, surge uma das diferenças entre a abordagem bayesiana e a frequentista: a forma como cada uma vê os parâmetros $\theta$ dos modelos (FERNANDES, 2009).

Segundo Brooks (2003) os frequentistas vêem o parâmetro $\theta$ como um valor fixo (não variável) e tentam estimar este valor desconhecido, por exemplo, maximizando uma função de verossimilhança. Os bayesianos também vêem os parâmetros como um valor fixo, porém por serem desconhecidos, são aleatórios e, por conseguinte, podem ser modelados por uma distribuição de probabilidades (ou função densidade de probabilidade - FDP) a qual resume o conhecimento que se tem sobre essas quantidades. Os bayesianos também acreditam que existe um valor verdadeiro para o parâmetro e utilizam sua correspondente distribuição a posteriori para obterem estimativas pontuais de um determinado parâmetro. Assim, à medida que o conhecimento sobre o parâmetro cresce, espera-se que a incerteza sobre ele diminua.

$\mathrm{Na}$ literatura técnica, são descritas diversas técnicas para avaliação da incerteza em modelos chuva-vazão, construídas sob o paradigma bayesiano. Entre estas, as técnicas de simulação e amostragem baseadas em métodos de Monte Carlo têm merecido especial atenção na literatura hidrológica (BEVEN; BINLEY, 1992 apud BEVEN, 2009; THIEMANN et al., 2001; WAGENER et al., 2003; MORADKHANI et al., 2005; VRUGT et al.,2011). Simulações de Monte Carlo são métodos de amostragem extremamente flexíveis e robustos, o que os torna aplicáveis a uma diversa gama de problemas. No caso dos modelos chuva-vazão, as principais vantagens estão associadas à inerente dificuldade em se desenvolver métodos analíticos que permitam lidar com a complexidade e não linearidade de múltiplas variáveis presentes na estrutura destes modelos.

Nas simulações de Monte Carlo, valores aleatórios de cada variável desconhecida (ex.: parâmetros $\theta$ ) são gerados de acordo com suas respectivas distribuições de probabilidade (a priori). Com isto, é possível obterem-se múltiplas realizações dos resultados do modelo (ex.: vazão associada a cada conjunto de $\theta$ ). Conhecendo-se uma realização do fenômeno modelado (ex.: vazão observada), e admitindo-se um critério para avaliação de sua similaridade em relação aos resultados obtidos via simulação (ex.: função de verossimilhança), é possível sumariar, através de propriedades estatísticas (ex.: moda, desvio padrão) e de uma distribuição de probabilidades empíricas (obtida a posteriori), o comportamento exibido pelas variáveis aleatórias e pelos resultados do modelo. A Figura 2 ilustra uma representação esquemática destes procedimentos.

Os métodos bayesianos aplicados à estimativa de incertezas de modelos hidrológicos combinam diversas variações de simulações de Monte Carlo com o teorema de Bayes, definido analiticamente como (BOX; TIAO, 1992):

$$
\begin{aligned}
& p(\theta \mid y)=\frac{p(y \mid \theta) \cdot p(\theta)}{p(y)}=c \cdot p(y \mid \theta) \cdot p(\theta) \\
& p(\theta \mid y) \propto L(\theta \mid y) \cdot p(\theta)
\end{aligned}
$$

na qual:

$\mathrm{p}(\theta)$ é a probabilidade da ocorrência de $\theta$;

$\mathrm{p}(\theta \mid \mathrm{y})$ é a probabilidade da ocorrência de $\theta$ dada a ocorrência de $y$;

$\mathrm{p}(\mathrm{y})$ e $\mathrm{p}(\mathrm{y} \mid \theta)$ são definidas analogamente;

$L(\theta \mid y)$ é denominada função de verossimilhança. 

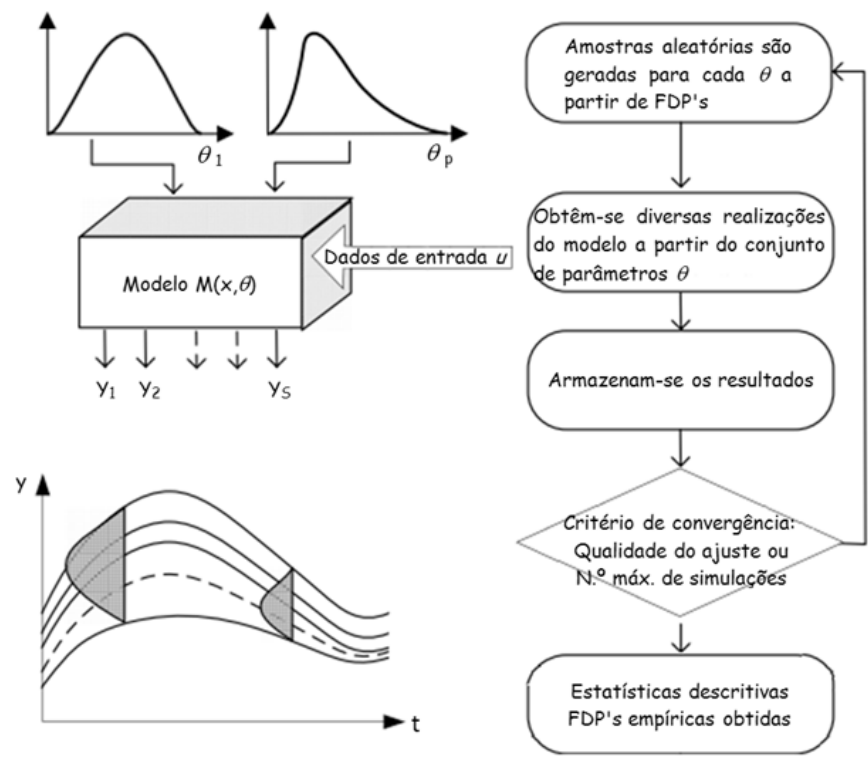

Figura 2 - Representação esquemática da simulação de Monte Carlo para análise de incertezas (adaptado de SHRESTHA, 2009)

A constante c é definida como:

$$
\begin{aligned}
& c^{-1}=p(y)=E[p(y \mid \theta)] \\
& c^{-1}=\left\{\begin{array}{l}
\int p(y \mid \theta) \cdot p(\theta) d \theta ; \theta \text { contínuo } \\
\sum p(y \mid \theta) \cdot p(\theta) ; \theta \text { discreto }
\end{array}\right.
\end{aligned}
$$

na qual a soma ou a integral é tomada em toda a gama de valores admissíveis de $\theta$ e $E[f(\theta)]$ é a esperança matemática de $\mathrm{f}(\theta)$ em relação à distribuição $\mathrm{p}(\theta)$.

$\mathrm{Na}$ expressão (1), $\mathrm{p}(\theta)$ é a distribuição a priori de $\theta$ e representa o que é conhecido a respeito de $\theta$ sem o prévio conhecimento de $\mathrm{y}$. De forma correspondente, $\mathrm{p}(\theta \mid \mathrm{y})$ reflete o conhecimento acerca dos parâmetros $\theta$ condicionado à ocorrência de y, isto é, a distribuição a posteriori de $\theta$. O fator c é simplesmente uma constante de normalização, necessária para garantir que a integral da distribuição a posteriori $\mathrm{p}(\theta \mid \mathrm{y})$ seja limitada à unidade.

Paulino et al. (2003) destacam que a função de verossimilhança $L(\theta \mid y)$ desempenha um importante papel no teorema de Bayes. É através dela que os dados observados $y$ "transformam" o conhecimento a priori sobre os parâmetros $\theta$. Em outras palavras, ela expressa a informação sobre $\theta$ fornecida pelos dados y. Segundo Box e Tiao (1992), a função de verossimilhança representa matematicamente uma forma do conhecimento prévio acerca do conjunto de parâmetros ser continuamente atualizado à medida que novas observações do fenômeno são coletadas. A Figura 3 ilustra uma representação esquemática deste conceito.

De fato, nos métodos de simulação de Monte Carlo empregados para avaliação bayesiana das incertezas em modelos chuva-vazão, a função de verossimilhança é empregada como

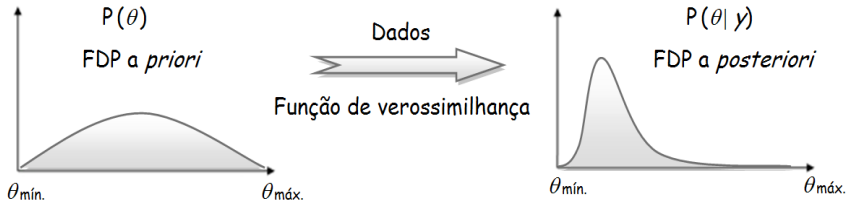

Figura 3 - Representação gráfica do teorema de Bayes (adaptado de KAVETSKI et al., data desconhecida)

critério para avaliação da convergência da simulação. O objetivo, via de regra, é maximizar (ou minimizar) seu valor a partir da comparação das séries simulada e observada. Para tal, é comum tomar-se o logaritmo natural da função para garantia que a mesma comporte-se de maneira monótona e estritamente crescente. Ademais, tendo em mente o teorema de Bayes, é importante observar que a aplicação do método está condicionada à admissão de um modelo probabilístico para os resíduos $r[\mathrm{p}(r \mid \theta)]$.

Ao se avaliarem estes métodos sobre outra perspectiva, notar-se-á que os parâmetros obtidos pela aplicação destas técnicas derivam-se de uma análise que busca essencialmente minimizar os resíduos do modelo. Por conseguinte, é comum observar na literatura técnica textos que tratam a estimativa de incertezas sob a perspectiva da calibração de modelos. A Figura 4 apresenta a relação entre estes tópicos.

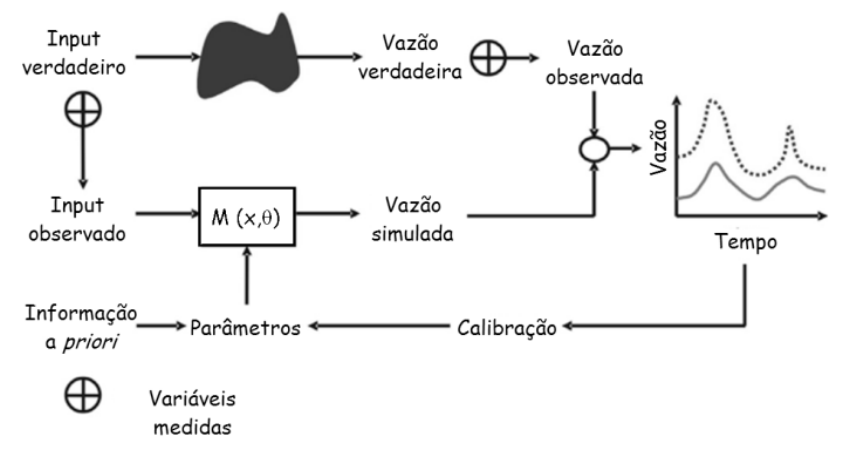

Figura 4 - Avaliação das incertezas sobre a perspectiva da calibração de modelos chuva-vazão (adaptado de VRUGT et al., 2008a)

Para que um modelo chuva-vazão seja útil em situações práticas, ele deve ser capaz de reproduzir as vazões observadas em uma seção fluviométrica de interesse, com suficiente grau de acurácia. Sob este cenário, admitindo-se que a estrutura conceitual do modelo seja capaz de representar o sistema hidrológico em análise, o que se busca na calibração é a identificação do conjunto de parâmetros $\theta$ que conduza à maior equivalência entre vazões observadas e simuladas.

Os algoritmos de calibração são formatações matemáticas de processos de tentativa e erro, segundo regras formais, que buscam reduzir os passos necessários para se atingir um objetivo pré-estabelecido, por exemplo, minimizar o erro quadrático de um modelo matemático por meio de estimativa de valores de parâmetros (EHR/UFMG, 2001). A qualidade da simulação é avaliada por critérios quantitativos (função objetivo), pela avaliação gráfica e pela análise de resíduos.

Ao se analisar o desenvolvimento destas técnicas nas 
últimas décadas, observar-se-á que as pesquisas enfocaram, sobretudo, os seguintes aspectos (VRUGT et al., 2013):

o desenvolvimento de funções objetivo que representassem de forma apropriada as diferenças entre vazões observadas e simuladas (resíduos);

a busca por algoritmos que pudessem solucionar de forma eficiente o problema da calibração;

a determinação da quantidade e tipo de informação necessária à aplicação dos métodos e avaliação dos resultados;

a seleção e o desenvolvimento de métodos numéricos capazes de solucionar os sistema de equações algébricas e diferenciais presentes nos modelos hidrológicos, de maneira eficiente e precisa;

a compreensão, a quantificação e a redução das incertezas; e

o desenvolvimento de técnicas que permitissem o refinamento contínuo (ou em 'tempo real') das soluções.

Não obstante os progressos realizados é importante destacar que a abordagem tradicional admite duas hipóteses muito fortes (VRUGT et al., 2013). A primeira está associada à premissa que os erros, presentes na conceitualização do fenômeno (estrutura do modelo) e nos dados observados (inputs u e output y), possam ser admitidos como desprezíveis e aleatórios. Em decorrência, presume-se que os resíduos da simulação exibirão um comportamento estatístico similar [ex.: $r \approx \mathrm{N}\left(0, \mathrm{~s}^{2}\right)$ ]. Há na literatura hidrológica uma série de trabalhos que demonstram que esta premissa é frequentemente violada. A segunda diz respeito ao critério utilizado como identificador de desempenho dos algoritmos de calibração. Independentemente dos fundamentos utilizados em sua definição, admitir que toda a informação contida nos dados observados possa ser extraída a partir de um indicador estatístico único (a ser maximizado ou minimizado) é inadequado. Por conseguinte, diferentes conjuntos de parâmetros conduzem a estatísticas muito similares, o que torna a escolha do "melhor" conjunto de parâmetros subjetiva. Beven e Binley (1992 apud BEVEN, 2009) utilizaram o termo "equifinalidade" para explicar esta subjetividade. Ademais, argumentaram que a super parametrização dos modelos hidrológicos, aliada à escassez e/ou baixa representatividade das informações hidrológicas utilizadas durante a calibração, justificariam este comportamento.

A utilização de métodos de simulação de Monte Carlo e técnicas de inferência bayesiana permite uma análise detalhada da primeira hipótese. Ademais, ao admitir que os parâmetros sejam variáveis aleatórias que seguem um modelo probabilístico, esta abordagem, senão corrige, relaxa parcialmente a segunda hipótese. Somente esta última é aqui analisada.

Este artigo concentra-se na avaliação das incertezas associadas às estimativas do conjunto de parâmetros $\theta$ de um modelo chuva-vazão conceitual, através do emprego de técnicas de inferência bayesiana via simulações de Monte Carlo. Esta avaliação considerou dois modelos probabilísticos, admitidos a priori, para os resíduos. As hipóteses admitidas pelos mesmos foram analisadas, sob a perspectiva de se obterem estimativas não enviesadas das incertezas.

Ressalta-se que esta pesquisa é parte integrante de outra maior, a qual além da compreensão e quantificação visa, sobretudo, a redução das incertezas associadas aos prognósticos de modelos chuva-vazão.

\section{METODOLOGIA}

Para o desenvolvimento dos estudos adotou-se a seguinte sequência metodológica:

identificação de uma bacia hidrográfica que possuísse monitoramento hidrológico adequado à proposta do estudo;

seleção de um modelo chuva-vazão de base conceitual;

escolha de um método de simulação de Monte Carlo para inferência bayesiana dos parâmetros do modelo;

análise dos resultados obtidos face aos modelos probabilísticos considerados para os resíduos: (i) avaliação dos modelos de erros; (ii) avaliação das incertezas associadas aos parâmetros estimados; e (iii) predição da incerteza na vazão modelada.

Nos subitens que se seguem as informações básicas e métodos utilizados são apresentados de maneira concisa. Os resultados obtidos são apresentados na próxima seção.

\section{Bacia hidrográfica e informações hidrológicas}

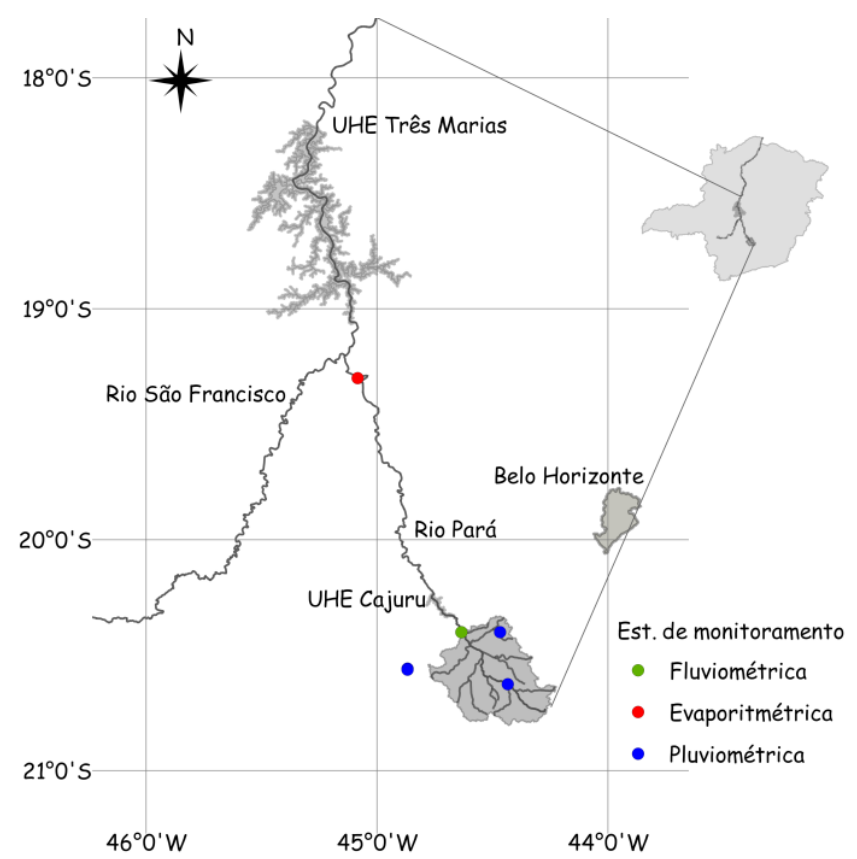

Figura 5 - Localização da bacia e estações de monitoramento analisadas (Datum: WGS84) 
O principal critério adotado para seleção da bacia hidrográfica foi a disponibilidade e pré-consistência das informações hidrológicas empregadas. Uma sub-bacia do Rio Pará (área de drenagem $\approx 1.620 \mathrm{~km}^{2}$; precipitação anual $\approx 1580 \mathrm{~mm}$ ), distante cerca de $90 \mathrm{~km}$ de Belo Horizonte, foi selecionada para o desenvolvimento dos estudos (Figura 5).

Na Tabela 1 é apresentada uma síntese das informações hidrológicas utilizadas. A escala temporal dos dados é diária. Destaca-se que estes dados foram previamente analisados por Lima (2004) e Fernandes (2006). As vazões e precipitações médias mensais características do período/bacia analisada são apresentadas, na sequência, por meio da Figura 6.

Tabela 1 - Informações hidrológicas utilizadas (escala temporal: diária)

\begin{tabular}{llll}
\hline Informação & $\begin{array}{l}\text { Código } \\
\text { ANA }\end{array}$ & $\begin{array}{l}\text { Entidade } \\
\text { responsável }\end{array}$ & $\begin{array}{l}\text { Período } \\
\text { analisado }\end{array}$ \\
\hline Evaporação & 1945017 & CEMIG & Calibração: \\
& 2044042 & ANA & $\begin{array}{l}\text { Out/1975 a } \\
\text { Set/1980 }\end{array}$ \\
Precipitação & 2044036 & ANA & Validação: \\
& 2044009 & CEMIG & Out/ 1980 a \\
& & & Set/1985 \\
\hline
\end{tabular}

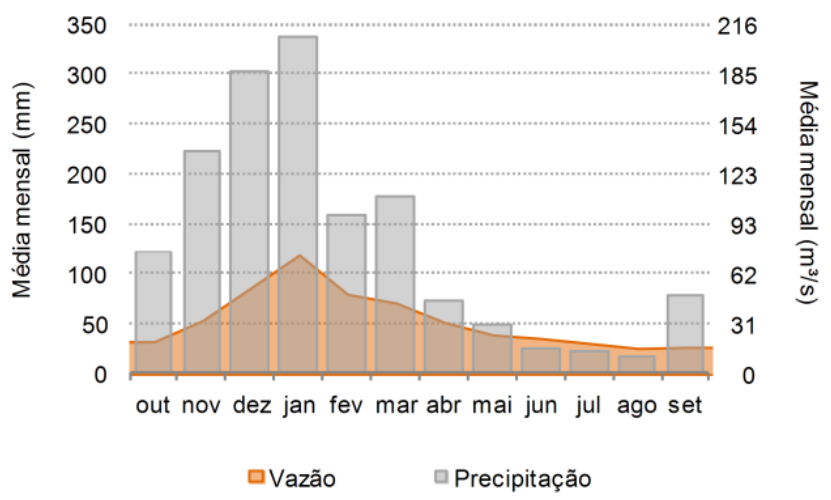

Figura 6 - Vazões e precipitações médias mensais no período/ bacia analisada

\section{Modelo chuva-vazão}

Os estudos utilizaram o modelo Rio Grande, desenvolvido pelo Departamento de Engenharia Hidráulica e Recursos Hídricos da UFMG (EHR/UFMG), com a finalidade primeira de controle operacional das usinas hidroelétricas da Companhia Energética de Minas Gerais (CEMIG) (EHR/UFMG, 2001). O Rio Grande compreende um modelo matemático completo das funções de produção e transferência do ciclo hidrológico, cujas equações procuram sintetizar as relações entre as variáveis do estado atual, permitindo o prognóstico dos estados futuros. Ademais, o modelo contempla um módulo de propagação de vazões, o que permite construir diferentes cenários de um sistema hidrológico. Por conseguinte, o modelo é classificado como conceitual, determinístico e semidistribuído (EHR/UFMG, 2001).

Neste modelo, a síntese determinística contínua da transformação chuva-vazão é realizada de modo concentrado, por meio de duas funções: a primeira, de produção, a qual executa a separação de um certo pulso de chuva bruta em deflúvio por unidade de tempo, enquanto a segunda, de concentração, faz a distribuição temporal dos pulsos de deflúvios, calculados em diferentes intervalos de tempo, concentrando-os e transformando-os em vazões na seção exutória.

A função de produção tem como base a estrutura do balanço de umidade do modelo conceitual Xinanjiang, inicialmente descrito por Zhao et al. (1980 apud ZHAO; LIU, 1995). Ela admite duas escalas temporais: horária e diária, sendo a última a empregada no estudo. A função de concentração utiliza o conceito do hidrograma unitário (HU) de Clark, na versão simplificada descrita por HEC (1981), a qual necessita apenas da especificação da forma predominante da bacia. A fim de preservar, ao menos em parte, a correção na representação temporal do processo de transformação da chuva e vazão, a função de concentração permite o emprego de uma duração temporal menor ou igual à utilizada pela função de produção. Para tal, os pulsos discretos de deflúvio total são uniformemente desagregados, segundo a duração admitida para o HU. As vazões obtidas, após a convolução, são regularizadas, segundo o intervalo de tempo dos dados empregados, através da média de $\mathrm{n}$ passos de cálculo, onde n é a razão entre os intervalos de tempo dos dados e do HU.

Tabela 2 - Parâmetros e intervalos de incerteza admitidos a priori para o modelo Rio Grande

\begin{tabular}{|c|c|c|c|}
\hline \multicolumn{2}{|c|}{ Parâmetros } & \multicolumn{2}{|c|}{ Limites } \\
\hline Símbolo & Descrição & Inferior & Superior \\
\hline $\mathrm{k}$ & Coeficiente de tanque & 0,55 & 1 \\
\hline imp & Fração de área impermeável & 0 & 0,03 \\
\hline wum & $\begin{array}{l}\text { Capacidade de tensão na zona } \\
\text { superior do solo }\end{array}$ & 5 & 150 \\
\hline wlm & $\begin{array}{l}\text { Capacidade de tensão na zona } \\
\text { inferior do solo }\end{array}$ & 50 & 250 \\
\hline wdm & $\begin{array}{l}\text { Capacidade de tensão na zona } \\
\text { profunda do solo }\end{array}$ & 5 & 150 \\
\hline sm & $\begin{array}{l}\text { Capacidade de água livre ou } \\
\text { gravitacional }\end{array}$ & 20 & 120 \\
\hline $\mathrm{b}$ & $\begin{array}{l}\text { Expoente da curva de } \\
\text { distribuição em área da } \\
\text { tensão capilar }\end{array}$ & 0,1 & 1 \\
\hline ex & $\begin{array}{l}\text { Expoente da curva de } \\
\text { distribuição espacial de } \\
\text { capacidade de água livre }\end{array}$ & 0,1 & 2 \\
\hline $\mathrm{c}$ & $\begin{array}{l}\text { Coeficiente de } \\
\text { evapotranspiração profunda }\end{array}$ & 0,01 & 0,4 \\
\hline kss & $\begin{array}{l}\text { Coeficiente do fluxo de } \\
\text { escoamento sub-superficial }\end{array}$ & 0,05 & 0,35 \\
\hline $\mathrm{kg}$ & $\begin{array}{l}\text { Coeficiente do fluxo de } \\
\text { escoamento subterrâneo }\end{array}$ & 0,05 & 0,65 \\
\hline ci & $\begin{array}{l}\text { Coeficiente de recessão do } \\
\text { fluxo sub-superficial }\end{array}$ & 0,1 & 0,99 \\
\hline $\operatorname{cg}$ & $\begin{array}{l}\text { Coeficiente de recessão do } \\
\text { fluxo subterrâneo }\end{array}$ & 0,8 & 0,999 \\
\hline
\end{tabular}

A estrutura do modelo assim construída necessita da calibração dos 13 parâmetros da função de produção. As incertezas associadas às estimativas do conjunto "ótimo" de 
parâmetros foram avaliadas, valendo-se de uma série de 5 anos tanto para calibração, quanto para validação (Tabela 1). Maiores detalhes acerca da estrutura da função de produção podem ser vistos em Zhao e Liu, 1995.

A Tabela 2 apresenta os parâmetros do modelo e os intervalos de incerteza admitidos a priori.

É válido destacar que para definição destes limites, diversas simulações foram realizadas. Para tal, tomaram-se como referência períodos na série fluviométrica onde se verificava extremos sazonais (vazões de pico e ao final da recessão). Ademais, foram consultadas publicações que se valeram da mesma função de produção (QUEIROGA, 2003; LIMA, 2004; LÜ et al., 2013; TIAN et al., 2013; ZHIJIA et al., 2013).

\section{Inferência bayesiana das incertezas dos parâme- tros via simulações de Monte Carlo}

O algoritmo selecionado para estimar a função densidade de probabilidade a posteriori dos parâmetros é denominado DREAM (Diffential Evolution Adaptive Metropolis). Este método de simulação de Monte Carlo via cadeias de Markov (MCMC) admite uma distribuição uniforme a priori para o conjunto de parâmetros $\theta$ e simula diferentes cadeias simultaneamente, ajustando a escala e forma do modelo distributivo proposto, durante a evolução da distribuição a posteriori (VRUGT et al., 2008a).

O método DREAM é uma modificação do método DE-MC (Differential Evolution Markov Chain) proposto por ter Braak (2006). Sua vantagem em relação a outros algoritmos reside, sobretudo, em sua eficiência, a qual decorre do ajuste automático da função proposta (SKAHILL, 2013). Destacase, que há na literatura uma série de referências à utilização do algoritmo para fins similares ao do presente artigo (HE et al., 2010; VRUGT; TER BRAAK, 2011; MINASNY et al., 2011; LALOY; VRUGT, 2012).

No tocante aos resíduos, foram analisadas 2 modelos probabilísticos: (i) o clássico (NL: normal likelihood), no qual presume-se que os erros da simulação apresentem um comportamento gaussiano $\left[\mathrm{r} \approx \mathrm{N}\left(0, \mathrm{~s}^{2}\right)\right.$; ; (ii) um generalizado (GL: generalized likelihood) (SCHOUPS, VRUGT, 2010), no qual admite-se que as diferenças entre vazões observadas e simuladas são correlacionadas, não estacionárias e probabilisticamente modeladas por uma distribuição exponencial potência assimétrica (SEP - Skew Exponential Power). As hipóteses admitidas por ambos os modelos foram verificadas a fim de garantir que a estimativa das incertezas nos parâmetros fosse não enviesada.

As funções de verossimilhança construídas segundo estes modelos introduzem parâmetros adicionais, os quais também devem ser estimados concomitantemente aos do modelo hidrológico analisado. Na Tabela 3 estes parâmetros estão apresentados. Ressalta-se que, a exemplo do modelo hidrológico, admitiu-se uma distribuição uniforme a priori para os mesmos.

Tabela 3 - Parâmetros e intervalos de incertezas admitidos a priori para os modelos dos resíduos

A descrição detalhada deste(s) método/modelos (de erro) foi preterida no presente artigo em detrimento à contextualização do tema e da análise dos resultados. Maiores detalhes acerca dos mesmos podem ser vistos em (VRUGT et al., 2008a; SCHOUPS; VRUGT, 2010).

\section{RESULTADOS E DISCUSSÃO}

A fim de compreender a influência de cada parâmetro sobre a qualidade do ajuste do modelo realizou-se inicialmente, uma análise de sensibilidade dos parâmetros do modelo Rio Grande. Esta avaliação revelou que os parâmetros k, wum, wlm, sm, kss, kg e ci impõem ao hidrograma modelado significativas variações quando da alteração de seus valores. Por conseguinte, as análises que se sucedem restringiram-se a esses parâmetros. É válido, no entanto, destacar que se observou um

\begin{tabular}{|c|c|c|c|c|}
\hline \multirow{2}{*}{ Mod. } & \multicolumn{2}{|c|}{ Parâmetros } & \multicolumn{2}{|c|}{ Limites } \\
\hline & Símbolo & Descrição & Inferior & Superior \\
\hline NL & $\sigma^{2}$ & Desvio padrão & 0 & 50 \\
\hline \multirow{5}{*}{ GL } & $\sigma_{0}^{2}$ & Heterocedasticidade: intercepção & -2 & 1 \\
\hline & $\sigma_{1}^{2}$ & Heterocedasticidade: inclinação & 0 & 1 \\
\hline & $\beta$ & Curtose & $-0,99$ & 1 \\
\hline & $\xi$ & Assimetria & 0,1 & 10 \\
\hline & $\phi_{1}$ & Coeficiente de autocorrelação & 0 & 1 \\
\hline
\end{tabular}

forte interação não-linear entre os parâmetros do modelo, o que produzia efeitos similares ou compensatórios sobre diferentes segmentos dos hidrogramas modelados, conforme a variação conjunta desses parâmetros.

\section{Avaliação dos modelos probabilísticos admitidos para os resíduos}

O primeiro modelo probabilístico analisado pressupôs que os erros do modelo eram independentes e identicamente distribuídos de acordo com a distribuição Normal $\left[\mathrm{N}\left(0, \mathrm{~s}^{2}\right)\right]$. Esta premissa, comumente admitida para explicar o comportamento dos resíduos na modelagem hidrológica, conduz o problema da estimação de parâmetros à abordagem sugerida pelo método dos mínimos quadrados padrão (SLS - standard least squares).

A Tabela 4 e a Figura 7 sumariam os resultados obtidos.

Tabela 4 - Estatísticas descritivas dos resultados admitindo-se a normalidade dos resíduos

\begin{tabular}{|c|c|c|c|}
\hline Estatística & Símbolo & Calibração & Validação \\
\hline Nash-Sutcliffe & NS & 0,871 & 0,797 \\
\hline Relação de volumes & RV & 0,988 & 1,023 \\
\hline $\begin{array}{l}\text { Raiz do erro quadrático } \\
\text { médio }\end{array}$ & RMSE & 10,2 & 14,8 \\
\hline C. de correl.. de Pearson & $\rho$ & 0,934 & 0,912 \\
\hline
\end{tabular}

Observa-se que o modelo foi capaz de reproduzir as vazões observadas com significativa acurácia, tanto no período de calibração (out/75 a set/80) quanto no de validação (out/80 a set/85), ainda que neste último as diferenças tenham sido ligeiramente acentuadas. 


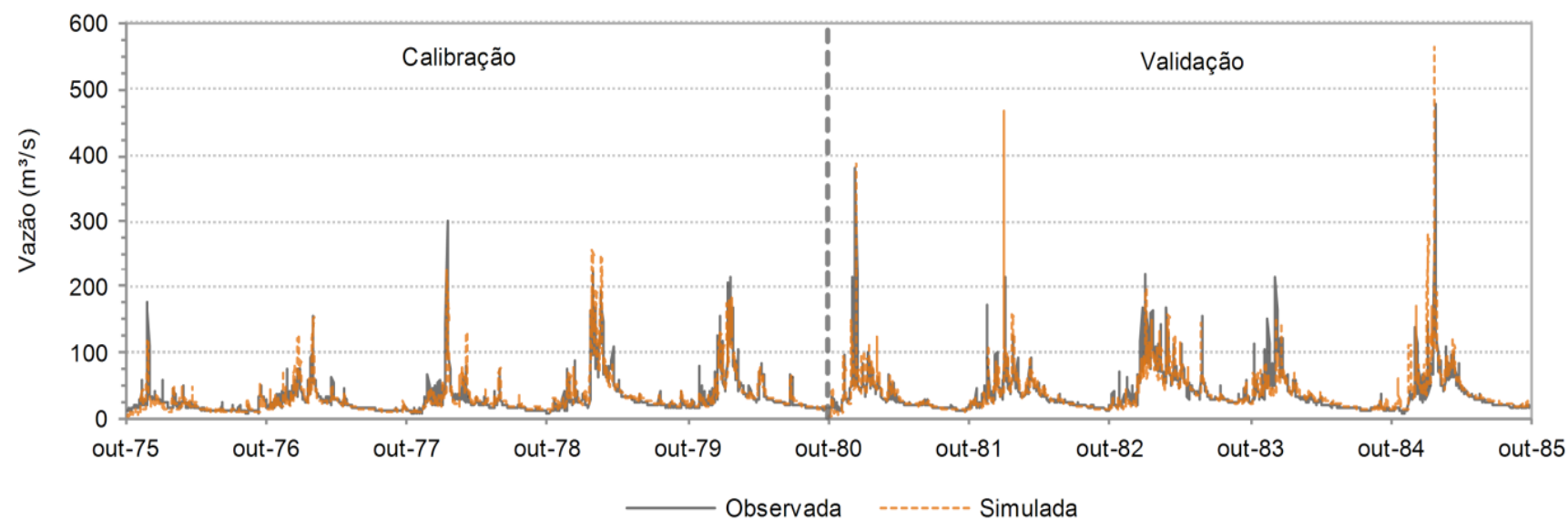

Figura 7 - Série temporal de vazões observadas e simuladas admitindo-se a normalidade dos resíduos

A análise dos resíduos, no entanto, revelou que os mesmos não seguem a hipótese de normalidade admitida a priori, conforme demonstrado pela Figura 8.

Neste quadro, evidencia-se que: (i) a variância aumenta com a magnitude da vazão simulada; e que (ii) os resíduos são significativamente autocorrelacionados. Isto contradiz as hipóteses admitidas de variância constante e de independência. Ademais, o confronto entre a distribuição dos resíduos e o modelo gaussiano corrobora para afirmação de que, apesar do elevado grau de similaridade entre vazões observadas e simuladas, o modelo de erros admitido é inadequado.

Outros estudos reportaram um comportamento dos resíduos análogo ao obtido (KUCZERA, 1983 apud SCHOUPS; VRUGT, 2010; THYER et al., 2009). Por ser uma hipótese frequentemente admitida (e violada), observou-se na última década um intenso debate na comunidade técnica acerca da especificação, desenvolvimento e avaliação (em uma abordagem formal ou informal) de funções de verossimilhança mais adequadas às técnicas de estimativas de incertezas na modelagem hidrológica construídas sob o paradigma bayesiano (BEVEN et al., 2008; VRUGT et al., 2008b).
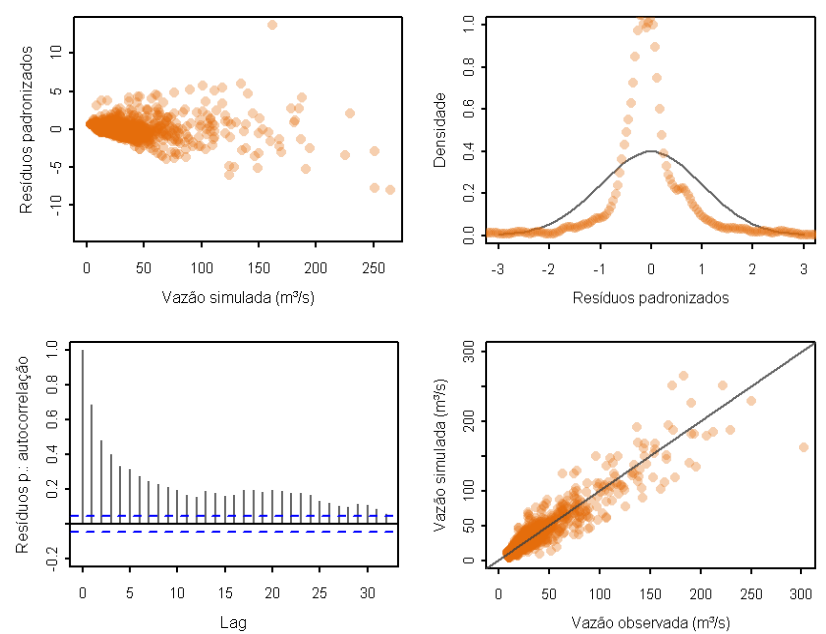

Figura 8 - Comportamento dos resíduos admitidos normais durante a calibração
O modelo generalizado (GL) de erros (SCHOUPS; VRUGT, 2010) insere-se neste contexto. Os resultados obtidos a partir de sua utilização estão sintetizados na Tabela 5 e na Figura 9.

Tabela 5 - Estatísticas descritivas dos resultados admitindo-se o modelo GL

\begin{tabular}{llrr}
\hline Estatística & Símbolo & Calibração & Validação \\
\hline Nash-Sutcliffe & NS & 0,848 & 0,813 \\
\hdashline \begin{tabular}{l} 
Relação de volumes \\
\hdashline $\begin{array}{l}\text { Raiz do erro quadrático } \\
\text { médio }\end{array}$
\end{tabular} & RV & 1,033 & 1,091 \\
\hdashline $\begin{array}{l}\text { Coeficiente de correlação } \\
\text { de Pearson }\end{array}$ & $\rho$ & 11,1 & 14,2 \\
\hline
\end{tabular}
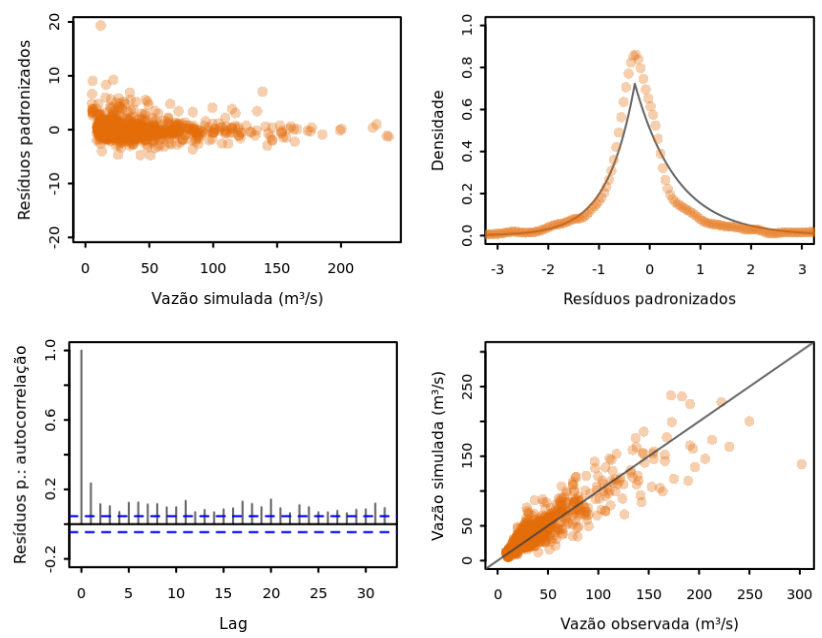

Figura 9 - Comportamento dos resíduos admitidos heterocedásticos, autocorrelacionados e modelados probabilisticamente segundo a FDP SEP durante a calibração

A exemplo do modelo de erros anterior, as estatísticas descritivas da qualidade do ajuste reportaram um elevado grau de similaridade entre vazões observadas e simuladas. Constata- 
se, entretanto, que o ajuste é levemente inferior ao observado anteriormente, ainda que a diferença entre os períodos de calibração e validação tenha sido atenuada. Em contrapartida, tanto a heterocedasticidade quanto a autocorrelação dos resíduos foram praticamente removidas (uma leve autocorrelação com lag 1 ainda é observada). A distribuição SEP ajustou-se muito bem à distribuição empírica dos resíduos, em especial, ao pronunciado pico observado em sua forma, o que a torna significativamente díspar da distribuição Normal.

A adoção do modelo generalizado de erros implica na necessidade de estimarem-se novos parâmetros simultaneamente aos do modelo hidrológico (resultados não apresentados). Apesar da maior complexidade o DREAM foi capaz de estimar estes parâmetros sem que tenham sido observados problemas de convergência. Exceção se faz ao parâmetro de autocorrelação f1, cuja presença representou uma elevação significativa no número de simulações necessárias à convergência do algoritmo. Por esta razão e a partir de uma análise gráfica dos resultados obtidos frente à sua variação, optou-se por fixá-lo $(0,70)$, a exemplo do parâmetro de curtose $\beta(1,0)$, que em função da forma exibida pela distribuição empírica dos resíduos tendia invariavelmente para a unidade.

O confronto entre as hipóteses admitidas pelas modelos de erros e os resíduos obtidos é essencial para obtenção de estimativas não enviesadas das incertezas. Há que se observar, entretanto, exemplos na literatura nos quais esta avaliação foi menosprezada (FEYEN et al., 2007). O decorrente impacto nas estimativas é destacado nos resultados descritos na sequência.

\section{Incertezas nas estimativas dos parâmetros}

As respectivas distribuições a posteriori dos parâmetros estimados foram obtidas para ambos os modelos de resíduos avaliados, ainda que o modelo NL tenha-se revelado inadequado. O objetivo é demonstrar o viés introduzido nos resultados. O comportamento exibido pelos parâmetros do modelo Rio Grande está apresentado na Figura 10.

É importante observar a expressiva alteração dos intervalos das estimativas dos parâmetros $\mathrm{k}$, wum e sm. A este respeito, é oportuno avaliar o viés introduzido na estimativa da incerteza. Considerando-se a análise de sensibilidade dos parâmetros e as informações hidrológicas utilizadas, pode-se inferir que:

k: há uma significativa incerteza nos dados de evaporação imposta, sobretudo, pela distância da estação evaporimétrica à bacia e pela dificuldade operacional no monitoramento da variável (tanque evaporimétrico). Por conseguinte, essa variação reflete um efeito compensatório da incerteza no dado de entrada (não considerada na análise) sobre a estimativa do parâmetro;

sm: sua alteração implica em uma variação pronunciada da magnitude das vazões de pico. A incerteza da estimativa refletida na diferença entre os modelos decorre da observação que o modelo GL enfatiza menos o ajuste das vazões de pico, devido à heterocedasticidade dos erros;
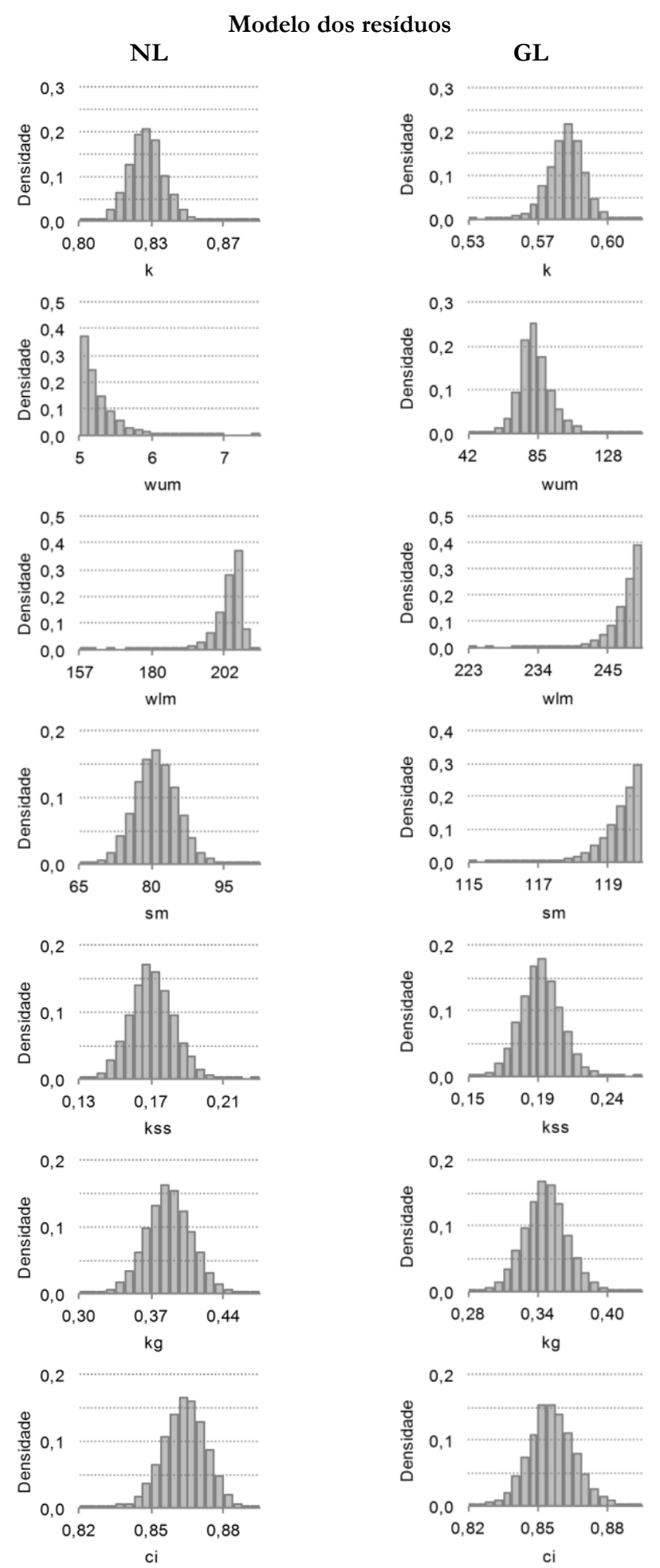

Figura 10 - Distribuições a posteriori dos parâmetros do modelo Rio Grande.

wum: ao efeito combinado da alteração das variáveis anteriores, as quais implicam necessariamente em variações no balanço de umidade do solo ou, em outras palavras, na maior ou menor disponibilidade de água para evapotranspiração e/ou geração de escoamento superficial. 
O maior padrão de regularidade exibido pelos demais parâmetros induz o modelador à crença de que o viés introduzido, neste caso, seja insignificante. No entanto, é fundamental, na discussão da adequação do modelo de erros e das incertezas nas estimativas nos parâmetros, se avaliar a diferença entre o comportamento exibido pela função de verossimilhança durante os períodos de calibração e validação. A Figura 11 ilustra a variação observada da função log de verossimilhança correspondente ao parâmetro $\mathrm{kg}$, sob os dois modelos de resíduos, para as fases de calibração e validação.

Durante a calibração, nota-se que a função de verossimilhança possui um valor máximo em ambos os modelos, ressaltando-se que este comportamento é mais explícito no modelo NL. Já na etapa de validação, na qual ambos os modelos apresentaram desempenho inferior à etapa de calibração (vide a diferença no valor máximo do logaritmo da função de verossimilhança), este comportamento somente é observado no modelo GL. Este fato, evidenciado também pela maior regularidade exibida nas estatísticas descritivas do modelo GL durante os períodos de calibração e validação, reafirma a adequação do modelo que pressupõe que os erros são heterocedásticos, autocorrelacionados e modelados probabilisticamente por uma FDP SEP.

Independentemente deste fato, é notório que diferentes estimativas do parâmetro conduzem a valores muito similares da função de verossimilhança. Em decorrência, a hipótese de um conjunto "ótimo", largamente difundida na literatura que trata da calibração de parâmetros sob a perspectiva de um problema de otimização, não pode ser verificada. De fato, há múltiplas estimativas "aceitáveis" dos parâmetros, as quais não podem ser rejeitadas facilmente. A tese da equifinalidade (BEVEN; BINLEY, 1992 apud BEVEN, 2009), confirmada na Figura 11, além de reiterar a importância de se avaliarem as incertezas associadas aos prognósticos dos modelos hidrológicos, reforça a forte influência da medida de verossimilhança sobre os resultados da modelagem.

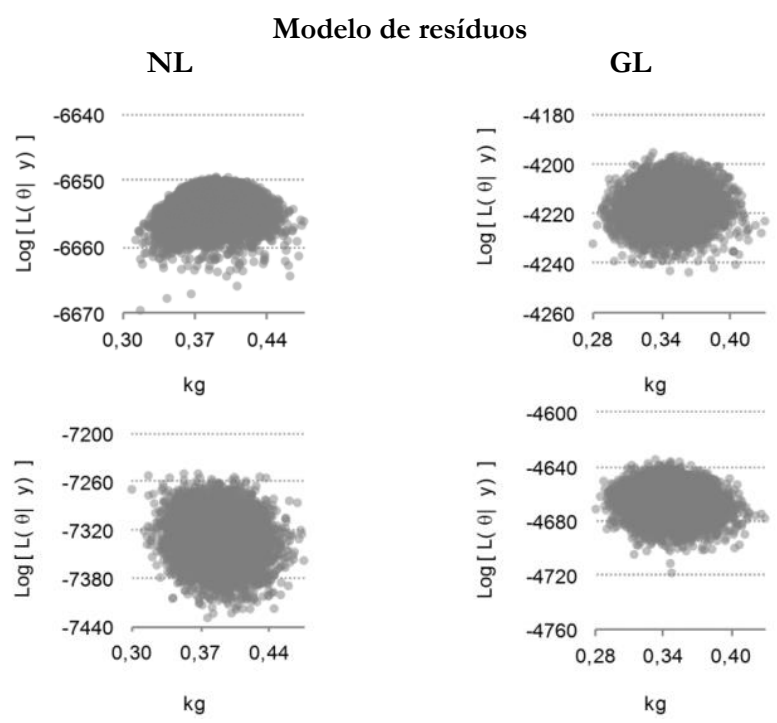

Figura 11 - Comportamento da função de verossimilhança para o parâmetro kg: calibração (acima) e validação (abaixo)

\section{Predição da incerteza na vazão modelada}

É possível, a partir da metodologia empregada, predizer a incerteza na vazão modelada. Os intervalos com 95\% de incerteza estão apresentados na Figura 12.

A inspeção do gráfico anterior revela que as vazões observadas durante o período de validação situam-se entre os limites calculados, ainda que, para algumas das vazões de pico observadas, o limite superior as tenha subestimado. Este fato indica, em uma primeira análise, que é necessária uma série mais extensa do que a utilizada para a calibração do modelo (5 anos). Não obstante esta hipótese, é necessário ponderar que a presente análise restringiu-se à avaliação das incertezas associadas aos parâmetros, desconsiderando as demais fontes (dados e estrutura do modelo), as quais podem representar outra importante parcela da incerteza na predição da vazão modelada.

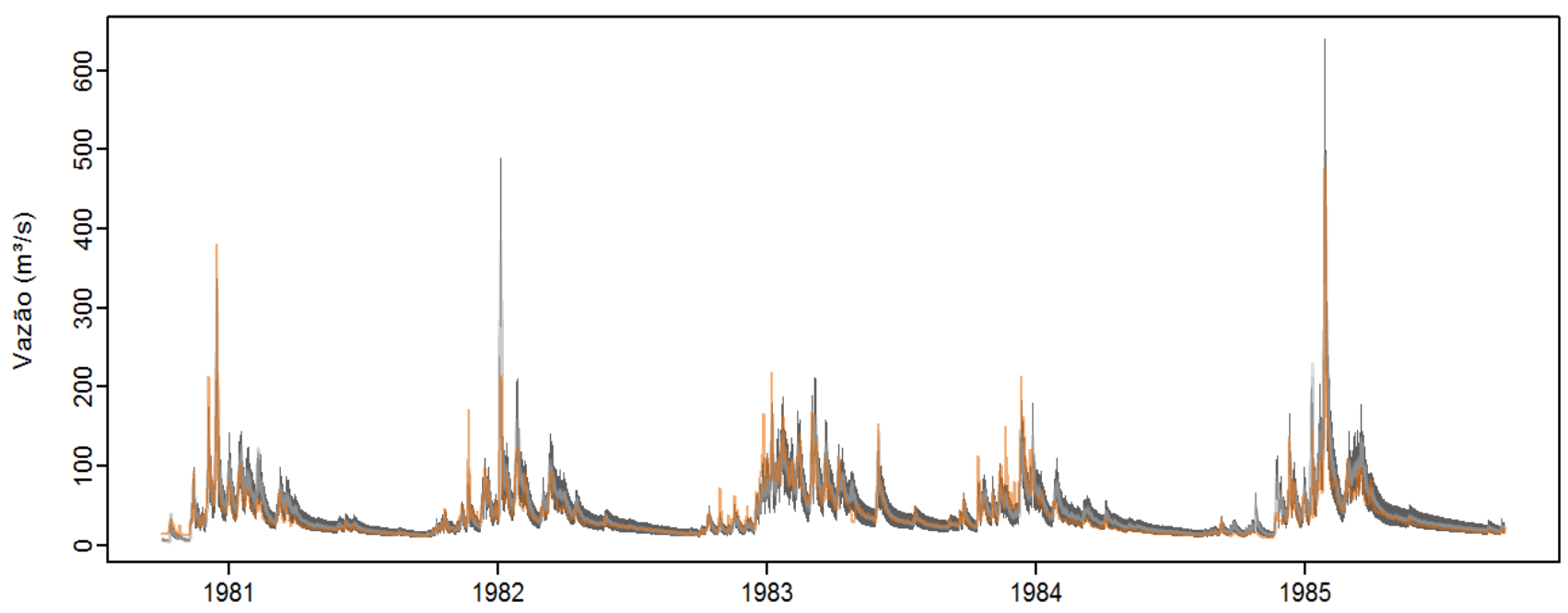

Figura 12 - Incerteza na predição da vazão modelada durante o período de validação utilizando-se o modelo de resíduos GL - série temporal observada (laranja) e intervalo com 95\% de incerteza: (i) decorrente das estimativas nos parâmetros (cinza claro); e (ii) total (cinza escuro) 


\section{CONSIDERAÇÕES FINAIS}

Este artigo avaliou as incertezas associadas às estimativas do conjunto de parâmetros $\theta$ de um modelo chuva-vazão conceitual, através do emprego de técnicas de inferência bayesiana via simulações de Monte Carlo. Neste estudo foram avaliados dois modelos probabilísticos para os resíduos. As hipóteses admitidas pelos mesmos foram analisadas e foi discutido o viés imposto às estimativas dos parâmetros.

A abordagem bayesiana revelou-se adequada ao objetivo do estudo. No entanto, é necessário ressaltar o impacto da medida de verossimilhança sobre os resultados obtidos. $\mathrm{O}$ modelo de resíduos generalizado GL foi capaz de explicar o comportamento admitido a priori para a diferença entre as vazões observadas e simuladas.

A incerteza na predição da vazão foi apresentada. O intervalo descrito foi capaz de representar as vazões observadas, apesar da subestimação de algumas vazões de pico. Esse fato enfatizou a necessidade de se avaliar a influência de outras fontes de incerteza sobre a vazão modelada.

Há que se destacar que o desconhecimento a priori da estrutura dos erros e a escassez de dados requerem, além da experiência do modelador, a integração de diferentes tipos de informações dentro de um quadro que é inevitavelmente subjetivo, uma vez que a própria informação é, muitas vezes, discutível (ex.: representatividade das observações de um pluviômetro face a um evento convectivo, a frequente escassez de medições de descarga líquida nos ramo superior das curvas de descarga, etc.). Não obstante estas dificuldades é crescente o consenso na comunidade técnica da necessidade de se avaliarem as incertezas envolvidas nos prognósticos dos modelos hidrológicos. O presente trabalho orientou-se por esta percepção, sob a perspectiva de contribuir para o avanço desta discussão, analisando um caso representativo da realidade brasileira.

\section{REFERÊNCIAS}

BEVEN, K. Environmental modelling: an uncertain future? Routledge, England: CRC Press, 2009.

BEVEN, K. Rainfall-runoff modeling: the primer. $2^{\text {nd }}$ ed. Chichester: John Wiley, , 2012.

BEVEN, K. \& BINLEY, A. The future of distributed models: model calibration and uncertainty prediction. Hydrological Processes, v. 6, n. 3, p. 279-298, July/Sept. 1992.

BEVEN, K.; SMITH, P. \& FREER, J. So just why would a modeller choose to be incoherent? Journal of Hydrology, v. 354, n. 1-4, p. 15-32, June 2008.

BOX, G. E. P. \& TIAO, G. C. Bayesian inference in statistical analysis. Chichester: John Wiley, 1992.
BROOKS, S. P. Bayesian computation: a statistical revolution. Philosophical Transactions of the Royal Society of London A, v. 361, n. 1813, p. 2681-2697, 2003.

BURNASH, R. J. C.; FERRAL, R. L.; MCGUIRE, R. A. \& MCGUIRE, R. A. A generalized streamflow simulation system: conceptual modeling for digital computers. California: U.S. Department of Commerce, National Weather Service and State of California, Department of Water Resources, 1973.

EHR/UFMG. Modelos de simulação hidrológica para previsão de vazões de curto prazo: relatório técnico parcial 1. Projeto de pesquisa aplicada. Belo Horizonte: EHR, 2001.

FERNANDES, W. Método para estimação de quantis de enchentes extremas com o emprego conjunto de análise bayesiana, de informações não sistemáticas e de distribuições limitadas superiormente. 2009. 185 f. Tese (Doutorado em Saneamento, Meio Ambiente e Recursos Hídricos) - Escola de Engenharia, Universidade Federal de Minas Gerais, Belo Horizonte, 2009.

FERNANDES, W. Metodologia unificada para análise de freqüência de vazões máximas anuais a partir da agregação da informação hidrometeorológica regionalizada. 2006. 145 f. Dissertação (Mestrado em Saneamento, Meio Ambiente e Recursos Hídricos) - Escola de Engenharia, Universidade Federal de Minas Gerais, Belo Horizonte, 2006.

FEYEN, L.;, VRUGT, J.; NUALLÁIN, B.; VAN DER KNIJFF, J. \& DE ROO, A. Parameter optimisation and uncertainty assessment for large-scale streamflow simulation with the LISFLOOD model. Journal of Hydrology, v. 332, n. 3-4, p. 276-289, Jan. 2007.

GONG, W.; GUPTA, H. V.; YANG, D.; SRICHARAN, K. \& HERO, A. O. Estimating epistemic and aleatory uncertainties during hydrologic modeling: An information theoretic approach. Water Resources Research, v. 49, n. 4, p. 2253-2273, Abr. 2013.

HE, M.; HOGUE, T. S.; FRANZ, K. J.; MARGULIS, S. A. \& VRUGT, J. A. Characterizing parameter sensitivity and uncertainty for a snow model across hydroclimatic regimes. Advances in Water Resources, v. 34, n. 1, p. 114-127, Jan. 2010.

HEC. Hydrologic Engineering Center. HEC-1 Flood Hydrograph Package - Reference Manual. Davis: U. S. Army Corps of Engineers, , 1981.

KAVETSKI, D.; KUCZERA, G.; THYER, M.; EVIN, G. \& RENARD, B. Uncertainties in flood forecasting and hydrological prediction: A bayesian total error 
perspective. [20--?]. 46 p. Apresentação em PDF. .

KUCZERA, G. Improved parameter inference in catchment models. Evaluating parameter uncertainty. Water Resources Research, v. 19, n. 5, p. 1151-1162, Oct. 1983.

KUCZERA, G.; KAVETSKI, D.; FRANKS, S. \& THYER, M. Towards a Bayesian total error analysis of conceptual rainfall-runoff models: characterising model error using storm-dependent parameters. Journal of Hydrology, v. 331, n. 1-2, p. 161-177, Nov. 2006.

KUCZERA, G. \& PARENT, E. Monte Carlo assessment of parameter uncertainty in conceptual catchment models: the Metropolis algorithm. Journal of Hydrology, v. 211, n. 1-4, p. 69-85, Nov. 1998.

LALOY, E.; VRUGT, J. High-dimensional posterior exploration of hydrologic models using multiple-try DREAM(ZS) and high-performance computing. Water Resources Research, v. 48, n. 1, W01526, Jan. 2012.

LIMA, A. Metodologia Integrada para Determinação da Enchente de Projeto de Estruturas Hidráulicas por Meio de Séries Sintéticas de Precipitação e Modelos Chuva-Vazão. 2004. 245 f. Dissertação (Mestrado em Saneamento, Meio Ambiente e Recursos Hídricos) - Escola de Engenharia, Universidade Federal de Minas Gerais, Belo Horizonte, 2004.

LINDSTROM, G.; JOHANNSON, B.; PERSSON, M.; GARDELIN, M. \& BERGSTROM, S. Development and test of the distributed HBV-96 hydrological model. Journal of Hydrology, v. 201, n. 1-4, p. 272-288, Dec. 1997.

LIU, Y. \& GUPTA, H. Uncertainty in hydrologic modeling: Toward an integrated data assimilation framework. Water Resources Research, v. 43, n. 7, July 2007.

LÜ, H.; HOU, T.; HORTON, R.; ZHU, Y.; CHEN, X.; JIA, Y.; WANG, W. \& FU, X. The streamflow estimation using the Xinanjiang rainfall runoff model and dual state-parameter estimation method. Journal of Hydrology, v. 480, p. 102-114, Feb. 2013.

MINASNY, B.; VRUGT, J. A. \& MCBRATNEY, A. B. Confronting uncertainty in model-based geostatistics using Markov Chain Monte Carlo simulation. Geoderma, v. 163, n. 3-4, p. 150-162, July 2011.

MONTANARI, A. Uncertainty of Hydrological Predictions. In: WILDERER, P. Treatise on Water Science., Oxford: Academic Press, 2011. cap. 2.17, v. 2, p. 459-478..

MOORE, R. The PDM rainfall-runoff model. Hydrology
E Earth System Sciences, v. 11, n. 1, p. 483-499, 2007.

MORADKHANI, H.; HSU, K.; GUPTA, H. \& SOROOSHIAN, S. Uncertainty assessment of hydrologic model states and parameters: Sequential data assimilation using the particle filter. Water Resources Research, v. 41, n. 5, p. 1-17, May 2005.

PAULINO, C.; TURKMAN, M. \& MURTEIRA, B. Estatística Bayesiana. Lisboa: Fundação Calouste Gulbenkian, 2003.

QUEIROGA, Y. G. A. Estudo e modelagem dos erros de simulação hidrológica e sua assimilação na previsão de vazões de curto prazo - o caso da bacia do rio Grande na UHE Camargos. 2003. 348 f. Dissertação (Mestrado em Saneamento, Meio Ambiente e Recursos Hídricos) - Escola de Engenharia, Universidade Federal de Minas Gerais, Belo Horizonte, 2003.

SCHOUPS, G. \& VRUGT, J. A formal likelihood function for parameter and predictive inference of hydrologic models with correlated, heteroscedastic, and nonGaussian errors. Water Resources Research, v. 10, n. 10, p. 1-17, Oct. 2010.

SHRESTHA, D. L. Uncertainty analysis in rainfall-runoff modelling: Application of machine learning techniques. 2009. Tese (Doutorado em Hidroinformática) UNESCO-IHE Institute for Water Education, Delft University of Technology, Netherlands, 2009.

SINGH, V. \& WOOLHISER, D. Mathematical Modeling of Watershed Hydrology. Journal of Hydrologic Engineering, v. 7, n. 4, p. 270-292, 2002.

SIRORSKA, A.; SCHEIDEGGER, A.; BANASIK, K. \& RIECKERMANN, J. Bayesian uncertainty assessment of flood predictions in ungauged urban basins for conceptual rainfall-runoff models. Hydrology and Earth System Sciences, v. 16, n. 4, p. 1221-1236, 2012.

SKAHILL, B. Practice driven and state of the art methods to quantify hydrologic model uncertainty. Vicksburg, MS: U.S. Army Engineer Research and Development Center, 2013. ERDC/CHL CHETN-IV-87.

SUN, S. \& BERTRAND-KRAJWESKI, J-L. Separately accounting for uncertainties in rainfall and runoff: Calibration of event-based conceptual hydrological models in small urban catchments using Bayesian method. Water Resources Research, v. 49, n. 9, p. 53815394, Sept. 2013.

ter BRAAK, C. A Markov Chain Monte Carlo version of the genetic algorithm Differential Evolution: easy Bayesian computing for real parameter spaces. Statistics 
and Computing, v. 16, n. 3, p. 239-249, Sept. 2006.

THIEMANN, T.; TROSSET, M.; GUPTA, H. \& SOROOSHIAN, S. Bayesian recursive parameter estimation for hydrologic models. Water Resources Research, v. 37, n. 10, p. 2521-2535, Oct. 2001.

THYER, M.; RENARD, B.; KAVETSKI, D.; KUCZERA, G.; FRANKS, S. \& SRIKANTHAN, S. Critical evaluation of parameter consistency and predictive uncertainty in hydrological modeling: A case study using Bayesian total error analysis. Water Resources Research, v. 45, n. 12, Dec. 2009.

TIAN, Y.; BOOIJ, M. \& XU, Y. Uncertainty in high and low flows due to model structure and parameter errors. Stochastic Environmental Research and Risk. Assessment, v. 28, n. 2, p. 319332, Feb. 2013.

VRUGT, J.; DIKS, C.; GUPTA, H.; BOUTEN, W. \& VERSTRATEN, J. Improved treatment of uncertainty in hydrologic modeling: Combining the strengths of global optimization and data assimilation. Water Resources Research, v. 41, n. 1, Jan. 2005.

VRUGT, J.; GUPTA, H. \& BOUTEN, SOROOSHIAN, S. A Shuffled Complex Evolution Metropolis algorithm for optimization and uncertainty assessment of hydrologic model parameters. Water Resources Research, v. 39, n. 8, 14p. 2003.

VRUGT., J.; ter BRAAK, C.; CLARK, M.; HYMAN, J. \& ROBINSON, B. Treatment of input uncertainty in hydrologic modeling: Doing hydrology backward with Markov chain Monte Carlo simulation. Water Resources Research, vol. 44, n. 12,15 p., 2008a.

VRUGT, J.; ter BRAAK, C.; DIKS, C. \& SCHOUPS, G. Hydrologic data assimilation using particle Markov chain Monte Carlo simulation: Theory, concepts and applications. Advances in Water Resources, v. 51, p. 457-478, 2013.

VRUGT, J. \& ter BRAAK, C. DREAM ${ }_{(\mathrm{D})}$ : an adaptive Markov Chain Monte Carlo simulation algorithm to solve discrete, noncontinuos, and combinatorial posterior parameter estimation problems. Hydrology and Earth System Sciences, v. 15, p. 3701-3713, 2011.

VRUGT, J.; ter BRAAK, C.; GUPTA, H. \& ROBINSON, B. Equifinality of formal (DREAM) and informal (GLUE) Bayesian approaches in hydrologic modeling? Stochastic Environmental Research and Risk Assessment, v. 23, n. 7, p. 1011-1026, 2008b.

WAGENER, T.; MCINTYRE, N.; LEES, M. \&WHEATER, H.; GUPTA, H. Towards reduced uncertainty in conceptual rainfall-runoff modeling: Dynamic identifiability analysis. Hydrological Processes, v. 17, n. 2, p. 455-476, 2003.
ZHAO, R. The Xinanjiang model applied in China. Journal of Hydrology, v. 135, n. 1-4, p. 371-381, 1992.

ZHAO, R. \& LIU, X. The Xinanjiang model. In: SINGH, V. (Ed.). Computer Models of Watershed Hydrology. Colorado, USA: Water Resources Publications, 1995. p. 215-232.

ZHAO, R.; ZHUANG, Y.; FANG, L.; LIU, X. \& ZHANG, Q. The Xinanjiang model. In: Hydrological Forecasting Proceedings Oxford Symposium, Wallingford, U.K.: International Association of Hydrological Sciences, 1980. p. 351-356. IAHS Publication n. ${ }^{\circ} 129$.

ZHIJIA, L.; PENGLEI, X. \& JIAHUI, T. Study of the Xinanjiang Model Parameter Calibration. Journal of Hydrologic Engineering, v. 18, n. 11, p. 1513-1521, Nov. 2013.

\section{Bayesian Evaluation of uncertainties in estimating the parameters of a conceptual rainfall-runoff mo- del.}

\section{ABSTRACT:}

This paper evaluated the uncertainties associated with the estimation of the parameters of a conceptual rainfall-runoff model, through the use of Bayesian inference techniques by Monte Carlo simulations. The Pará River sub-basin, located in the Upper São Francisco River basin, was selected for developing the studies. In this paper, we used the Rio Grande conceptual bydrologic model (EHR / UFMG, 2001) and the Markov Chain Monte Carlo simulation method named DREAM (VRUGT, 2008a). The results showed that the Bayesian approach proved to be adequate to the proposed objectives, enabling and reinforcing the importance of evaluating the uncertainties associated with the results simulated by bydrological models.

Keywords: Rainfall-runoff modeling; Uncertainty assessment; Bayesian inference; Rio Grande model; DREAM. 
Avaliação bayesiana das incertezas nas estimativas dos parâmetros de um modelo chuva-vazão conceitual 